\title{
Macroscale superlubricity achieved via hydroxylated hexagonal boron nitride nanosheets with ionic liquid at steel/steel interface
}

\author{
Zhiwen ZHENG ${ }^{1,2}$, Xiaolong LIU ${ }^{1}$, Guowei HUANG ${ }^{3}$, Haijie CHEN $^{1,2}$, Hongxiang $\mathbf{Y U}^{1,2}$, Dapeng FENG ${ }^{1, *}$, \\ Dan QIAO ${ }^{1, *}$ \\ ${ }^{1}$ State Key Laboratory of Solid Lubrication, Lanzhou Institute of Chemical Physics, Chinese Academy of Sciences, Lanzhou 730000 , China \\ ${ }^{2}$ University of Chinese Academy of Sciences, Beijing 100049, China \\ ${ }^{3}$ State Key Laboratory of Advanced Processing and Recycling of Nonferrous Metals, Lanzhou University of Technology, Lanzhou 730050, \\ China
}

Received: 10 May 2021 / Revised: 18 June 2021 / Accepted: 25 July 2021

(C) The author(s) 2021.

\begin{abstract}
Macroscale superlubricity is a prospective strategy in modern tribology to dramatically reduce friction and wear of mechanical equipment; however, it is mainly studied for point-to-surface contact or special friction pairs in experiments. In this study, a robust macroscale superlubricity for point-to-point contact on a steel interface was achieved for the first time by using hydroxylated modified boron nitride nanosheets with proton-type ionic liquids (ILs) as additives in ethylene glycol aqueous $\left(\mathrm{EG}_{\mathrm{aq}}\right.$ ). The detailed superlubricity process and mechanism were revealed by theoretical calculations and segmented experiments. The results indicate that hydration originating from hydrated ions can significantly reduce the shear stress of $\mathrm{EG}_{\mathrm{aq}}$, which plays an essential role in achieving superlubricity. Moreover, the IL induces a tribochemical reaction to form a friction-protective film. Hydroxylated boron nitride nanosheets (HO-BNNs) function as a polishing and self-repairing agent to disperse the contact stress between friction pairs. Superlubricity involves the change in lubrication state from boundary lubrication to mixed lubrication. This finding can remarkably extend the application of superlubricity for point-to-point contact on steel surfaces for engineering applications.
\end{abstract}

Keywords: macroscale superlubricity; ionic liquid (IL); hydroxylated boron nitride; steel/steel interface; mixed lubrication

\section{Introduction}

Friction and wear of machinery and equipment are inevitable phenomena that cause a significant loss of energy and equipment damage [1]. The most effective method is the use of lubricants to avoid direct contact between friction pairs [2,3]. Given recent innovative progress in reducing friction and wear, superlubricity, defined as a friction coefficient (COF) of less than 0.01, is considered a viable strategy [4]. Nowadays, superlubricity, including solid superlubricity [5] and liquid superlubricity [6], has attracted continuously attention in many fields including machinery, energy, aerospace, and biology [7-10]. As for solid superlubricity, the achievement of extremely low COF (<0.01) usually relied on a specific structure (e.g., graphite, $\mathrm{MoS}_{2}$, a-C:H, and diamond like carbon (DLC) film) [11-15] or a certain atmosphere (e.g., inert gas and vacuum) [16-18]. However, for liquid superlubricity, it is much easier to obtain common friction pairs in the atmosphere; therefore, it has wider application. However, various kinds of oils fail to realize superlubricity because of their high pressure-Viscosity coefficients [19, 20]. Evidently, green water-based lubricants possess unparalleled advantages in the superlubricity field because of their appropriate

* Corresponding authors: Dapeng FENG, E-mail: dpfeng@licp.cas.cn; Dan QIAO, E-mail: ddqiao@licp.cas.cn 
pressure-viscosity coefficients [21].

In recent years, numerous studies on water-based superlubricity systems have expanded the application of superlubricity and our understanding of its mechanism [22, 23]. Initially, Water lubrication on ceramic surfaces has been systematically investigated by Kato's group [24, 25] and Klein's group [26, 27], and an ultralow COF (0.002-0.007) was achieved after the running-in period. The superlubricity mechanism was attributed to the boundary lubrication layer of colloidal silica generated by the tribochemical reaction between the ceramic surfaces and water. Meanwhile, smoother contact surfaces were formed after the running-in period. Subsequently, the lubrication behavior of water and polyol systems has been investigated in prior studies [28, 29]. They reported that the COF between glass and ceramic or ceramic and ceramic can decrease to 0.004 , which arises from hydrated ions generated by acid or a mixture of polyol and acid. The mechanism of superlubricity was attributed to the formation of a hydrogen ion adsorption layer and an elastohydrodynamic film with extremely low shear strength. The tribochemical reaction also played a pivotal role in achieving superlubricity. Ge et al. [30] utilized the induced tribochemical reaction to situ-form ionic liquids (ILs) on ceramic interfaces and achieved ultralow COF (0.003) and excellent antiwear properties. Other studies on liquid superlubricity have been reported, including the aqueous lubrication of charged polymer brushes [31], in situ formation of tribofilm with MXene nanoflakes in glycerol, the synergistic effect of graphene-oxide nanoflakes and ethanediol [32], IL-modified carbon quantum dots (CQDs) [33], two-dimensional nanoadditives [6], and macromolecular structures [34].

The aforementioned studies on liquid superlubricity focused on ceramic friction pairs under low applied loads, and the contact forms of friction were mainly point-to-surface, line-to-surface, and surface-to-surface $[35,36]$. By contrast, the point-to-point contact form was considered difficult in achieving superlubricity owing to the extremely high contact stress. In actual engineering applications, the normal mechanical equipment mainly consists of steelwork components, and is employed in high-load conditions [3]. However, macroscale superlubricity for point-to-point contacts at the steel interface has not yet been reported. Therefore, it is of great significance to develop new lubrication systems to implement the application of superlubricity in engineering applications.

In this study, a feasible superlubricity system was designed for the first time for steel/steel contact in the point-to-point form (at $196 \mathrm{~N}$, corresponding to 1.81 GPa, Table S1 in the Electronic Supplementary Material (ESM)). On the basis of the superlubricity mechanism of aqueous lubrication, which mainly refers to the hydration effect that reduces the shear strength of the lubricant and the running-in process to decrease the contact pressure, and aqueous ethylene glycol ( $\left.E_{\mathrm{aq}}\right)$ was selected as the base lubricant because of its excellent anticorrosion and sustainability. A proton-type IL with a long alkyl chain was adopted as the hydrated ion to decrease the shear strength of $E_{\mathrm{aq}}$ [37]. Meanwhile, hydroxylated boron nitride nanosheets (HO-BNNs) with uniform dispersion were used to accelerate the running-in period [38]. The discovery of this lubrication system will greatly promote the application of superlubricity to point-to-point contact at the bearing steel interface.

\section{Experimental}

\subsection{Materials}

Boron nitride (BN) powders $(1 \mu \mathrm{m}, 98.5 \%)$ and $\mathrm{N}, \mathrm{N}$-dimethyldodecylamine (98\%) were supplied by Shanghai Macklin Biochemical Co., Ltd., China. Bibutyl phosphate (98\%) was purchased from Sa En Chemical Technology Shanghai Co., Ltd., China. The other reagents used are analytical reagent (AR). 1,3-propylene glycol, and 1,4-butylene glycol were obtained from Tianjin Ke Miou Chemical Reagent Co., Ltd. Glycerol was obtained from Tianjin Kaitong Chemical Reagent Co., Ltd., China. The other chemicals used were of analytical grade and were used without further purification. Deionized water was prepared by the Lanzhou Institute of Chemical Physics, Chinese Academy of Sciences, China.

\subsection{Preparation of proton-type IL}

A proton-type IL, N,N-dimethyldodecan-1-aminium dibutyl phosphate $\left(\mathrm{DMAP}_{4}\right)$, was synthesized by 
proton transfer reactions between phosphates and alkanolamines, as shown in Fig. S1 in the ESM. Briefly, equimolar dibutyl phosphate and $\mathrm{N}, \mathrm{N}-$ dimethyldodecylamine were added to a round-bottom flask containing $30 \mathrm{~mL}$ acetonitrile, purified three times with nitrogen, and heated in an oil bath at $65{ }^{\circ} \mathrm{C}$ for $12 \mathrm{~h}$. Then, the solvents were removed by distillation under reduced pressure and washed several times with anhydrous ether to obtain a colorless oily liquid. The structures of the IL were determined by the nuclear magnetic resonance (NMR, Bruker Avance III $400 \mathrm{MHz}$ spectrometer, Switzerland) and the high resolution mass spectrometry (HRMS, Bruker micrOTOF Q II, Switzerland). The results are presented in the ESM 1.1 and corresponding spectra are showed in Figs. S2 and $\mathrm{S} 3$ in the ESM.

\subsection{Preparation of HO-BNNs}

HO-BNNs were prepared by ultrasonic peeling of commercial BN powders $(1 \mu \mathrm{m})$. Figure $\mathrm{S} 4$ in the ESM shows a schematic illustration of the synthesis process of HO-BNNS and a photo image of the HOBNNs aqueous solution. In brief, BN powder $(1.0 \mathrm{~g})$ was added to $100 \mathrm{~mL}$ of a glass beaker holding $50 \mathrm{~mL}$ of deionized water. Then, the beaker was subjected to ultrasonic treatment $(120 \mathrm{~W})$ for $48 \mathrm{~h}$. In this process, hydroxyl functional groups can be introduced between the freshly stripped $\mathrm{BN}$ sheets with a number of activated dangling bonds. After ultrasonic treatment, the predispersion was further treated by centrifugation for $30 \mathrm{~min}$ at 2,000 $\mathrm{rpm}$, and the supernatant was collected and denoted as HO-BNNs.

\subsection{Preparation of lubricant samples}

Water-based lubricant samples were prepared by mixing $50 \mathrm{wt} \%$ ethylene glycol (EG) and $50 \mathrm{wt} \%$ deionized water (denoted as $\mathrm{EG}_{\mathrm{aq}}$ ). $\mathrm{DMAP}_{4}$ was used as an additive in the as-prepared $\mathrm{EG}_{\mathrm{aq}}$ with mass ratios of $0.5 \%, 1 \%, 1.5 \%, 2 \%$, and $2.5 \%$, which were denoted as IL-EG $\mathrm{Eq}_{\mathrm{aq}}$. Likewise, a certain mass of HO-BNNs aqueous solution was added to IL-EG $\mathrm{Fq}_{\mathrm{aq}}$ to obtain lubricant samples with $\mathrm{HO}-\mathrm{BNN}$ s mass concentrations of $20,35,50,65$, and $80 \mu \mathrm{g} / \mathrm{mL}$ (denoted as HO-BNNs/ IL-EG $\left.G_{a q}\right)$. To further expand the lubrication system, three types of polyhydric alcohols [1,3-propylene glycol (PG), 1,4-butylene glycol (BG), and glycerine (GI)] were used as solvents to study the lubrication performance of water-based lubricants after adding $1.5 \mathrm{wt} \%$ IL and $65 \mu \mathrm{g} / \mathrm{mL}$ HO-BNNs. The obtained suspensions were denoted as $\mathrm{HO}-\mathrm{BNNs} / \mathrm{IL}-\mathrm{PG}_{\mathrm{aq}}, \mathrm{HO}-\mathrm{BNNs} / \mathrm{IL}-\mathrm{BG}_{\mathrm{aq}}$ and $\mathrm{HO}-\mathrm{BNNs} / \mathrm{IL}-\mathrm{GI}_{\mathrm{aq}}$. Before the tribological test, all lubricant samples were sonicated for $2 \mathrm{~min}$ to obtain a uniform dispersion.

\subsection{Friction test}

The friction experiments were conducted on a fourball tribometer (MS-10 A, China, accuracy of $10 \mathrm{mN}$ ) to assess the lubrication performance of the water-based samples. The corresponding description of the fourball tribometer is shown in Fig. S5 in the EMS. The test conditions were a rotary speed of 1,200 r/min and a constant load of $196 \mathrm{~N}$ for 30 min under steel-steel contact (GCr 15; $\phi=12.7 \mathrm{~mm}$, HRC: 64-66, $R_{\mathrm{a}} \approx 10 \mathrm{~nm}$ ). In addition, a variable speed ranges from 600 to 1,450 $\mathrm{r} / \mathrm{min}$ and the variable load range from 98 to $245 \mathrm{~N}$ experiments were tested. Table S1 in the ESM shows the initial contact stresses and sliding linear velocities corresponding to different loads and rotary speeds, respectively. All tests were carried out at $25 \pm 3{ }^{\circ} \mathrm{C}$, $30 \%-45 \% \mathrm{RH}$, and repeated three times to ensure dependability and precision.

\subsection{Characterizations}

The morphology of the HO-BNNs was characterized by the atomic force microscope (AFM; MultiMode 8-HR, Germany) and the high-resolution transmission electron microscope (HRTEM; JEM 2100F, Japan). The chemical components, groups, and electron binding energies were analyzed by the Fourier transform infrared spectroscope (FTIR; Thermo Fisher Scientific, Germany), the Raman spectroscope (LabRAM, HR Evolution, France), and the X-ray photoelectron spectrometer (XPS; ULVAC-PHI PHI 5000 Versaprobe III, Japan). The crystal structure of the HO-BNNs was analyzed using the X-ray diffraction (XRD; Malvern Panalytical Empyrean, UK). The thermal stabilities of the HO-BNNs and IL were tested via the simultaneous thermogravimeter (NETZSCH STA 449 F3 Jupiter, Germany). The stability of HO-BNNs/IL-EG aq was measured using the UV-Vis spectrophotometer (Hitachi U-3900, Japan ). In addition, the density and kinematic viscosity of the lubricant samples were measured using 
a SYP1003-III kinematic viscometer. After the friction tests, the lower three balls were cleaned, and the wear scar diameters (WSDs) of the wear scars were measured using a color imaging microscope (XDS-0745D, China). The morphologies of the worn regions were characterized by a scanning electron microscope (SEM; JSM-5600LV, Japan) and a 3D profiler (MicroXAM-800, USA). The chemical properties of the worn surface after rubbing were analyzed using the XPS (ULVACPHI PHI 5000 Versaprobe III, Japan). Furthermore, a detailed characterization of the inside of the worn surface was obtained using the focused ion beam transmission electron microscope (FIB-TEM; FEI Strata 400S, USA).

\section{Results and discussion}

\subsection{Characterization of HO-BNNs}

The TEM image (Fig. 1(a)) reveals that the as-prepared HO-BNNs have a roughly uniform size and shape. The HRTEM image (Fig. 1(b)) indicates that the lattice distance between adjacent fringes was approximately $0.27 \mathrm{~nm}$ (the inset in Fig. $1\left(\mathrm{~b}_{\mathrm{ii}}\right)$ ), which could be ascribed to the (110) plane of the BN crystal (PDF\#50-1,504). Furthermore, the AFM analysis (Fig. 1(c)) showed that the size of HO-BNNs was approximately $0.9-1.5 \mu \mathrm{m}$, and the thickness was approximately $5 \mathrm{~nm}$. The Raman spectra (Fig. 1(d)) indicate that there was a slight blue shift in the peak positions of HO-BNNs $\left(1,371.6 \mathrm{~cm}^{-1}\right)$
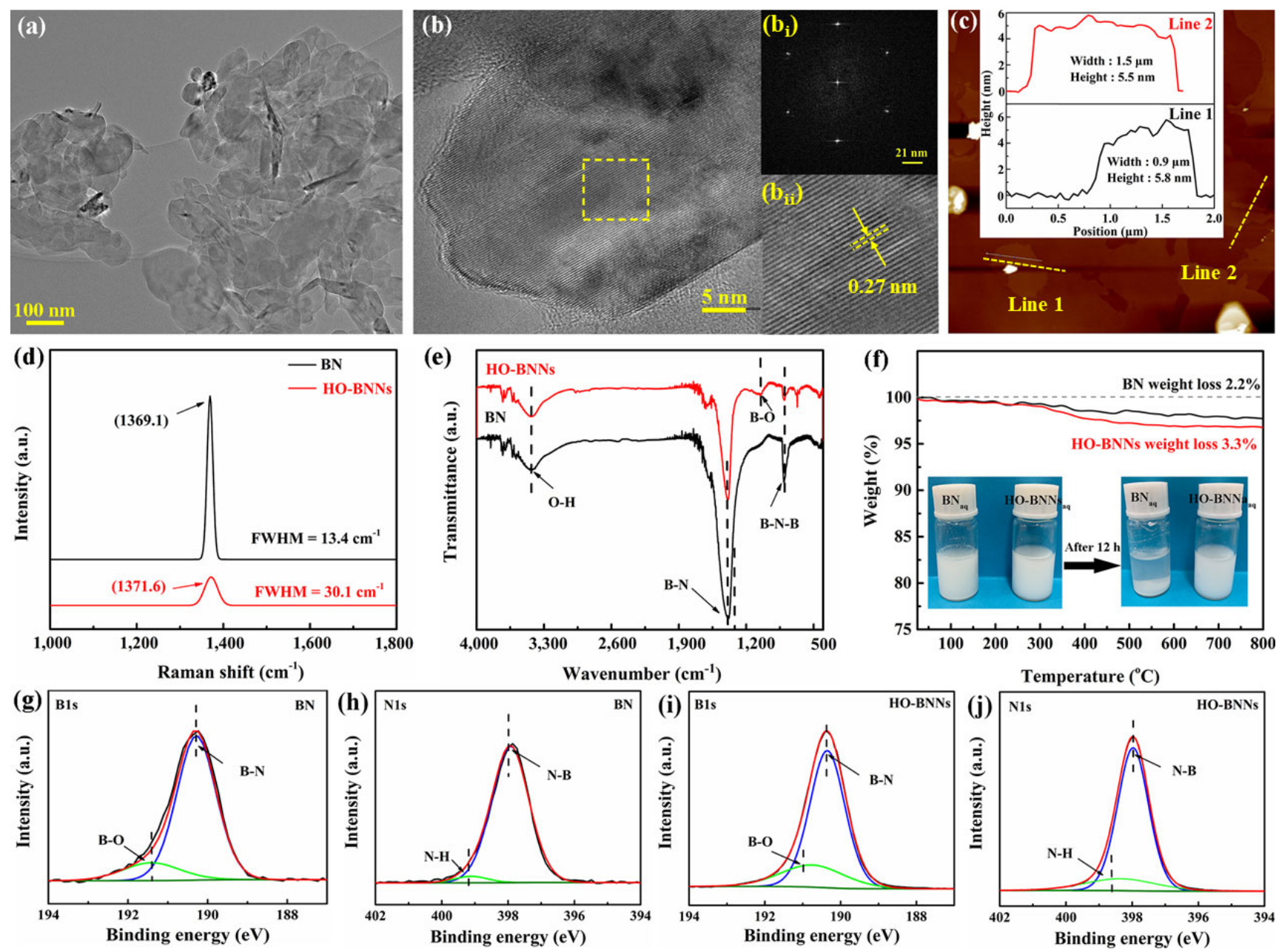

Fig. 1 Microstructure characterization and chemical composition of BN and HO-BNNs: (a) low-magnification and (b) high-magnification TEM images of HO-BNNs (insets: $\left(b_{i}\right)$ is corresponding selected area electron diffraction pattern and $\left(b_{i i}\right)$ is lattice spacing). (c) AFM image of as-prepared HO-BNNs and corresponding height profile, (d) Roman pattern, (e) FTIR spectra, (f) TGA curves of pristine $\mathrm{BN}$ and $\mathrm{HO}-\mathrm{BNNs}$, and $(\mathrm{g}-\mathrm{j}) \mathrm{XPS}$ spectra of $\mathrm{BN}$ and $\mathrm{HO}-\mathrm{BNNs}$ fitted $\mathrm{B} 1 \mathrm{~s}$ and $\mathrm{N} 1 \mathrm{~s}$. 
compared with the initial BN powder $\left(1,369.1 \mathrm{~cm}^{-1}\right)$. The full width at half maxima (FWHM) of HO-BNNs increased from 13.4 to $30.1 \mathrm{~cm}^{-1}$, which occurred because HO-BNNs have a higher in-plane strain level and lower interlaminar interaction leading to the softening of phonons $[39,40]$.

The FTIR spectra (Fig. 1(e)) showed two strong absorption peaks at 1,386 and $803 \mathrm{~cm}^{-1}$, which correspond to the in-plane $\mathrm{B}-\mathrm{N}$ stretching vibration of $\mathrm{sp}^{2}$-bonded and out-of-plane $\mathrm{B}-\mathrm{N}-\mathrm{B}$ bending vibrations. After exfoliation, a new absorption peak appeared at $1,050 \mathrm{~cm}^{-1}$, corresponding to the symmetric B-O stretching band [41, 42]. The thermogravimetric analysis (TGA) curves of pristine $\mathrm{BN}$ and $\mathrm{HO}-\mathrm{BNNs}$ (Fig. 1(f)) indicate that the final weight loss of BN at $800{ }^{\circ} \mathrm{C}$ was approximately $2.2 \%$, which is lower than that of HO-BNNs (3.3\%). Meanwhile, the optical images of the newly configured $\mathrm{BN}$ and HO-BNNs aqueous solution and after 12-h standing are inserted in Fig. 1(f), which revealed that HO-BNNS had better dispersion stability because of the increase in hydroxyl groups. The XPS spectra of pristine $\mathrm{BN}$ and HO-BNNs are shown in Figs. $1(\mathrm{~g})-1(\mathrm{j})$. The B 1s spectra could be fitted into two peaks at 190.3 and $191.4 \mathrm{eV}$, which can be attributed to the $\mathrm{B}-\mathrm{N}$ and $\mathrm{B}-\mathrm{O}$ bonds, respectively. $\mathrm{N} 1$ s could be fitted into two peaks at 397.9 and $398.6 \mathrm{eV}$, corresponding to $\mathrm{N}-\mathrm{B}$ and $\mathrm{N}-\mathrm{H}$, respectively [43]. On the basis of the aforedescribed analysis, it can be concluded that the hydroxyl group was successfully modified onto the surface edge of the BNNs.

\subsection{Characterization of lubrication samples}

Four lubrication samples $\left(\mathrm{EG}_{\mathrm{aq}}, \mathrm{BN}-\mathrm{EG}_{\mathrm{aq}}, \mathrm{HO}-\mathrm{BNNs}-\right.$ $\mathrm{EG}_{\mathrm{aq}}$, and $\mathrm{HO}-\mathrm{BNNs} / \mathrm{IL}-\mathrm{EG}_{\mathrm{aq}}$ ) were prepared by ultrasonication, and their basic physical characteristics were evaluated. Figure 2(a) shows the wettability of the four lubrication samples on the bearing steel surface. After adding the HO-BNNs, the surface tension of the $\mathrm{EG}_{\mathrm{aq}}$ increased. However, IL can significantly reduce the surface tension of $\mathrm{EG}_{\mathrm{aq}}$ appearing as a small contact angle and leading to the steel surface being completely wetted. Figure 2(b) displays the visible images of four samples in fresh and setting for five days. The results showed that HO-BNNs/IL-EGaq exhibited the best stability without obvious precipitation. Meanwhile, for $B N-E_{a q}$ and $H O-B N N s-E_{a q}$, significant precipitation

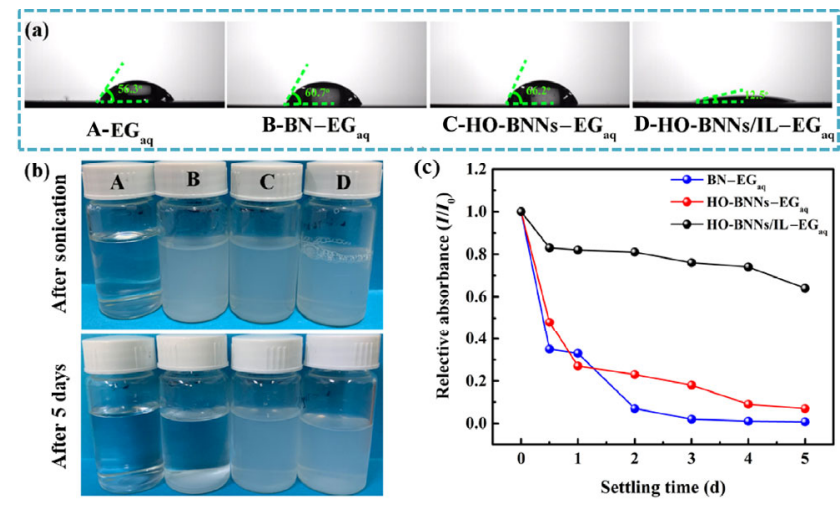

Fig. 2 (a) Contact angles of lubricant samples droplets on bearing steel surface (A: $E_{a q}, B: B N-E G_{a q}, C: H O-B N N s-E_{a q}$, and D: HO-BNNs/IL-EG $\mathrm{Eq}_{\mathrm{aq}}$ ), (b) digital images of four dispersions at different settling time, (c) relative absorbance $\left(I / I_{0}\right)$ of $\mathrm{BN}-\mathrm{EG}_{\mathrm{aq}}$, $\mathrm{HO}-\mathrm{BNNs}-\mathrm{EG}_{\mathrm{aq}}$, and $\mathrm{HO}-\mathrm{BNNs} / \mathrm{IL}-\mathrm{EG}_{\mathrm{aq}}$ studied by UV-Vis spectra using $\mathrm{EG}_{\mathrm{aq}}$ as blank.

appeared after standing for five days. UV-Vis measurements were carried out to further evaluate the dispersion stability of the solution (Fig. 2(c)). Compared with $B N-E G_{a q}$ and $\mathrm{HO}-B N N s-E G_{a q}$, the relative absorbance of the $\mathrm{HO}-\mathrm{BNNs} / \mathrm{IL}-\mathrm{EG}_{\mathrm{aq}}$ remained at 0.65 after setting for 5 days, illustrating that IL can increase the dispersion stability of HO-BNNs.

Additionally, the density and viscosity of the four samples at room temperature were measured, as shown in Table S2 in the ESM. Their densities were similar because of the small amounts of additives. The HO-BNNs/IL-EG aq sample had the smallest kinematic viscosity of the samples.

\subsection{Friction test}

Figure 3(a) shows the evolution of COF with time lubricated by pure water, EG, EG $\mathrm{aq}_{\mathrm{a}}$ HO-BNNs-EG $\mathrm{Bq}_{\text {, }}$ IL-EG ${ }_{\mathrm{aq}}$, and HO-BNNs/IL-EG ${ }_{\mathrm{aq}}$. The COF of pure water was maintained at approximately 0.34 , while the COFs of EG aq and HO-BNNs-EG $E_{\mathrm{aq}}$ fluctuated between 0.10 and 0.33 , which occurred because the water vapor formed by friction heat has an important influence on the friction process. For example, when only pure water is used as a lubricant, the temperature of the oil tank is extremely high after rubbing because of poor lubrication performance, which generates water vapor to form a gas, liquid, and solid three-phase interface (water vapor, water, and steel). This causes severe fluctuations in the COF. 

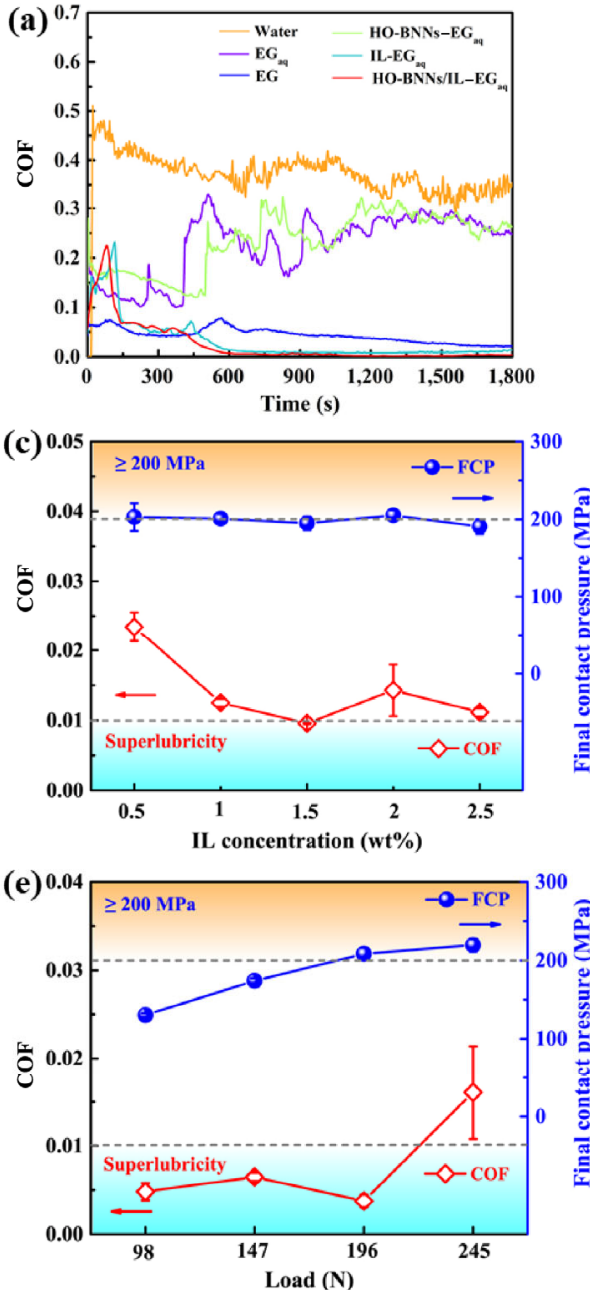
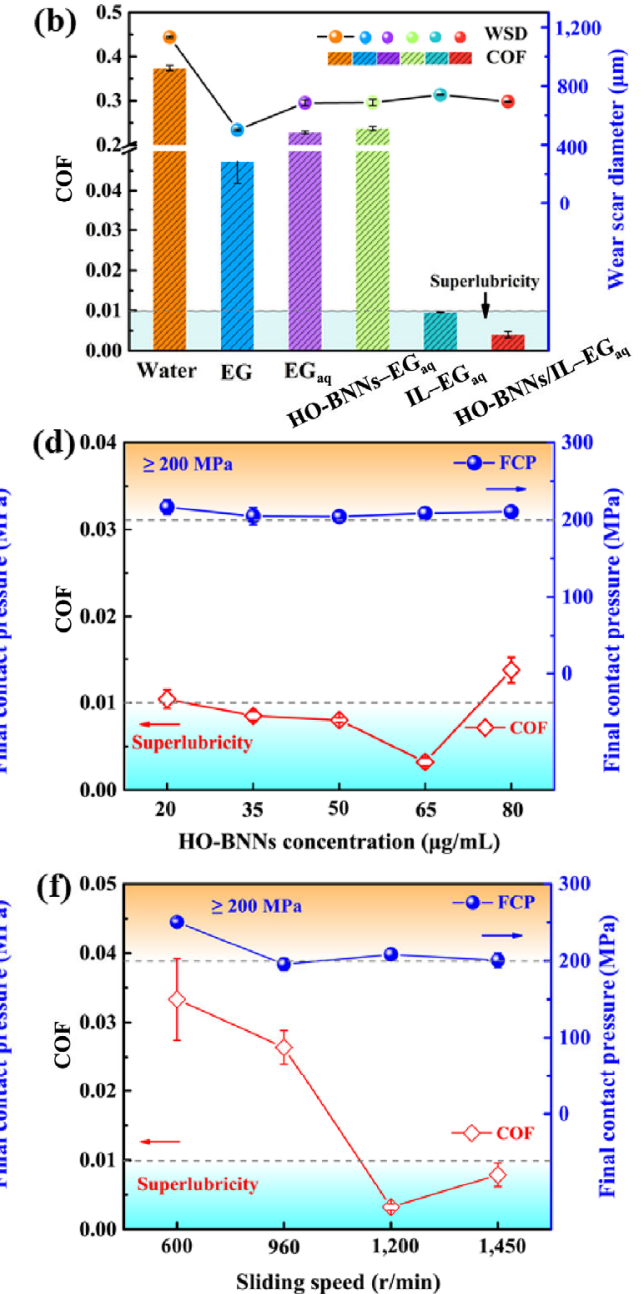

Fig. 3 Characterizations of the liquid superlubricity under $196 \mathrm{~N}, 1,200 \mathrm{r} / \mathrm{min}$. (a) Variations of COF curves with time, (b) average

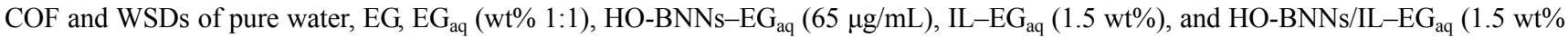
IL and $65 \mu \mathrm{g} / \mathrm{mL}$ HO-BNNs), (c) different IL concentrations in $\mathrm{EG}_{\mathrm{aq}}$, (d) different HO-BNNs concentrations in IL-EG $\mathrm{aq}_{\text {, }}$ (e) different loads lubricated by $\mathrm{HO}-\mathrm{BNNs} / \mathrm{IL}-\mathrm{EG}_{\mathrm{aq}}(1,200 \mathrm{r} / \mathrm{min})$, and (f) different sliding speeds lubricated by HO-BNNs/IL-EG $\mathrm{Eq}(196 \mathrm{~N})$.

When using $\mathrm{EG}_{\mathrm{aq}}$ or HO-BNNs-EG $\mathrm{aq}_{\mathrm{aq}}$ as lubricants, the elevated temperature at the friction interface causes the temperature of the oil tank to rise sharply, and water is constantly evaporated, during which the COF oscillates at approximately 400-1,000 s. After 1,000 s, the water almost completely evaporated, leaving only the higher viscosity glycol; thus, the COF was relatively stable. However, higher temperatures will cause the EG to polymerize to form a higher viscosity, resulting in a higher COF than pure EG. The polymerization of EG can be confirmed by the higher viscosity of the liquid remaining in the oil box after friction. Moreover, the single EG had a lower COF, approaching 0.045 (Fig. 3(a)). Figure 3(b) shows the averages of the steady-state COF (after a running-in period of approximately $600 \mathrm{~s}$ ) and the corresponding WSDs. The optical photographs of the wear regions are shown in Fig. S6 in the ESM. The average WSD lubricated by EG was $505 \mu \mathrm{m}$, which was the smallest of all WSDs.

Then, proton-type IL $\left(\mathrm{DMAP}_{4}\right)$ with a long alkyl chain was adopted as an additive to reduce COF. After adding $1.5 \mathrm{wt} \%$ IL to $\mathrm{EG}_{\mathrm{aq}}$ the COF curve (Fig. 3(a)) first experienced a serious running-in period (approximately $180 \mathrm{~s}, \mathrm{COF}$ up to 0.2 ). The COF suddenly decreased to approximately 0.07 and gradually dropped to about 0.02 at approximately $1,000 \mathrm{~s}$ and finally fluctuated in the range of 0.02-0.009. This shows that the superlubricity state was achieved. After adding IL to $E_{a q}$, the temperature of the oil box did not increase during the entire friction process, which is attributed to the 
excellent antifriction performance of the IL. Therefore, there is almost no change in the viscosity of the lubricant because there was almost no evaporation of water. A stable lubrication system ensures superlubricity. However, for other concentrations of IL, although the COFs were significantly reduced, the superlubricity state was not achieved (Fig. 3(c)). The decrease in COF may arise from the formation of a thick and dense lubricating film to reduce the contact of friction pairs, and the contact stress decreases as the wear scar increases. In addition, the final contact pressure (FCP) was approximately $200 \mathrm{MPa}$, which can be calculated using Eq. (S1) in the ESM by assigning WSD.

Based on the optimal concentration of IL, HO-BNNs were selected to further decrease the COF. When $65 \mu \mathrm{g} / \mathrm{mL}$ HO-BNNs were added, the lowest COF (0.004) was obtained. The superlubricity state can be achieved with 35-65 $\mu \mathrm{g} / \mathrm{mL}$ HO-BNNs (Fig. 3(d)). When the concentration of HO-BNNs was increased to $85 \mu \mathrm{g} / \mathrm{mL}$, the COF (0.014) jumped out of the superlubricity region. This is because moderate amounts of HO-BNNs can be well dispersed and enter the friction contact area to assist lubrication, and excessive HOBNNs are easily entangled and aggregated with each other, which restricts the HO-BNNs from entering the friction contact region.

The influence of the loads and sliding speeds were investigated, as depicted in Figs. 3(e) and 3(f), respectively. It was observed that the $\mathrm{COF}$ fluctuated in a small range of $0.003-0.006$ at a load of less than $196 \mathrm{~N}$. When the load increased to $247 \mathrm{~N}$, the superlubricity state was not observed throughout the experiment, and the COF increased to 0.016. Meanwhile, when the sliding speeds decreased to 960 and $600 \mathrm{r} / \mathrm{min}$, and the corresponding COFs increased to 0.028 and 0.033 , respectively. Subsequently, the sliding speed increased to $1,450 \mathrm{rpm}$, and the COF value increased from 0.003 to 0.008 . Therefore, a stable superlubricity state can be achieved in a suitable load range $(<250 \mathrm{~N})$ and a sliding speed range $(>1,200 \mathrm{~m} / \mathrm{s})$. Furthermore, after static settlement for two weeks, the superlubricity state was still obtained with a COF value of 0.009 (Fig. 4(a)). To explore the macroscale superlubricity of other polyols in this system, $\mathrm{HO}-\mathrm{BNNs} / \mathrm{IL}-\mathrm{PG}_{\mathrm{aq}}$ HO-BNNs/IL-BG ${ }_{\mathrm{aq}}$ and HO-BNNs/IL-GI ${ }_{\mathrm{aq}}$ suspensions as lubricants were tested, and the corresponding friction curves are shown in Fig. 4(b). Superlubricity behaviors were observed after a certain running-in period, which indicated that this superlubricity system had a wider engineering application.

\subsection{Characterization of wear scars and debris}

After the friction tests, the wear scars of the lower balls were characterized using SEM and 3D micrographs. For the basic $E_{\mathrm{aq}}$, the worn surface exhibited severe scuffing with a large volume of flakings and many deep grooves. The corresponding WSD $(789.6 \mu \mathrm{m})$ was the largest (Fig. S6 in the ESM). When employing a single IL as the additive of $\mathrm{EG}_{\mathrm{aq}}$, the WSD decreased to $740.4 \mu \mathrm{m}$ and the worn surface developed multiple wide furrows and small flakes (Figs. 5(a) and 5(b)). The wear debris collected from the wear surfaces indicated that there were some iron filings with sizes in the range of 30-200 nm (Fig. S7(a) in the ESM). These hard, large abrasive particles filled the surface of the steel balls during friction, causing severe abrasive wear.
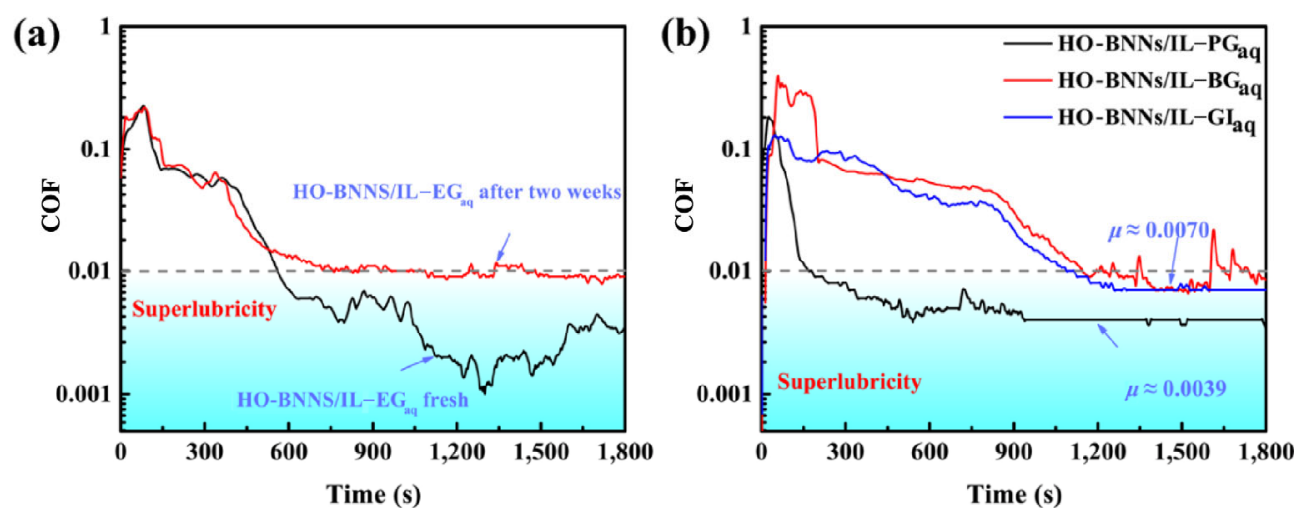

Fig. 4 (a) COF curves of HO-BNNs/IL-EG $\mathrm{Eq}_{\mathrm{aq}}$ suspensions: fresh and after two weeks, (b) variation of COFs of HO-BNNs/IL-PG $\mathrm{HO}-\mathrm{BNNs} / \mathrm{IL}-\mathrm{BG}_{\mathrm{aq}}$, and $\mathrm{HO}-\mathrm{BNNs} / \mathrm{IL}-\mathrm{GI}_{\mathrm{aq}}$ suspensions under $196 \mathrm{~N}$ and 1,200 r/min. 

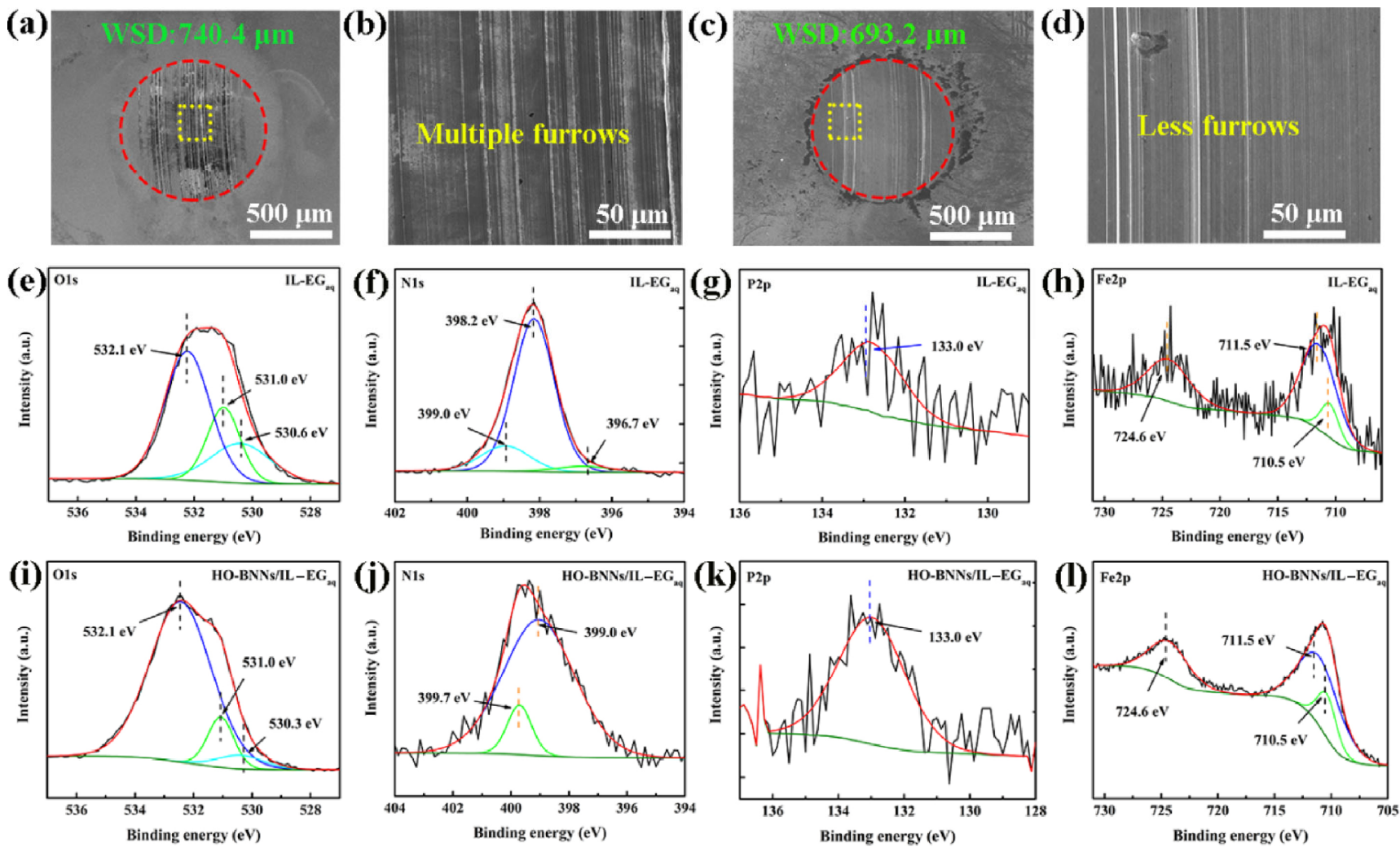

Fig. 5 SEM images of wear scars lubricated by (a, b) IL-EG $\mathrm{Eq}_{\mathrm{aq}}$ and (c, d) HO-BNNs/IL-EG $\mathrm{Eq}_{\mathrm{aq}}$ under the conditions of $196 \mathrm{~N}, 1,200 \mathrm{r} / \mathrm{min}$, and $1,800 \mathrm{~s}$. Corresponding XPS spectrum of wear scars lubricated by (e-h) IL-EG $\mathrm{Eq}_{\mathrm{aq}}$ and (i-1) HO-BNNs/IL-EG $\mathrm{E}_{\mathrm{aq}}$.

After adding HO-BNNs, the WSD further decreased to $693.2 \mu \mathrm{m}$ and the worn surface presented large area of flatness with less shallows, narrow wear scars, and small pits (Figs. 5(c) and 5(d)), which may be attributed to the surface polishing and self-repairing effects of HO-BNNs. The wear debris collected from the wear surfaces was composed of amorphous $\mathrm{BN}$ and iron filings with sizes in the range of 65-235 $\mathrm{nm}$ (Fig. S7(d) in the ESM).

XPS is an effective method for explaining the lubrication mechanism by detecting the elemental composition and chemical state of the wear surface. Figures 5(e)-5(l) show the XPS spectra of O 1s, N 1s, $\mathrm{P} 2 \mathrm{p}$, and Fe $2 \mathrm{p}$ of the worn surfaces lubricated by IL-EG ${ }_{\text {aq }}$ and HO-BNNs/IL-EG aq. The peaks of O $1 \mathrm{~s}$ appeared at 532.1, 531.0, and $530.6 \mathrm{eV}$, which can be assigned to the bond of iron oxides, hydroxides, and phosphorus oxide, respectively. The binding energies of $\mathrm{N}$ 1s of lubricated by IL-EG ${ }_{\mathrm{aq}}$ were located at 399.0, 398.2, and $396.7 \mathrm{eV}$, which were ascribed to nitrides and organic nitrogen compounds. The peaks of $\mathrm{N} 1 \mathrm{~s}$ lubricated by HO-BNNs/IL-EG ${ }_{\text {aq }}$ appeared at 399.7 and
$399.0 \mathrm{eV}$, and they represented residual HO-BNNs and nitrides [44], respectively, which confirmed the self-repairing effect of HO-BNNs. The peak of the $\mathrm{P} 2 \mathrm{p}$ spectrum was $133.0 \mathrm{eV}$, corresponding to organic phosphates. Moreover, $\mathrm{Fe}_{2} \mathrm{O}_{3}, \mathrm{FeOOH}$, and $\mathrm{Fe}_{3} \mathrm{O}_{4}$ were identified from the peaks of 724.6, 711.5, and $710.5 \mathrm{eV}$, respectively, in the spectrum of Fe $2 \mathrm{p}$ [45]. A tribochemical reaction film composed of iron oxide, nitride, and phosphide was formed on the wear surface. This reduced wear by preventing direct contact between the friction pairs.

To further seek the direct evidence of the tribofilm established on the worn surfaces lubricated by HO-BNNs/IL-EG ${ }_{a q}$, FIB-TEM characterization was introduced to explore the microstructure and chemical composition of tribofilm. Figure 6(a) verifies that a continuous and uniform tribofilm covered the surface of the meal matrix, where some small wear debris and the accumulation marked by yellow rectangles could be detected. Closer inspection revealed that the thickness of tribofilm at the platform was about $3.23 \mathrm{~nm}$ (Fig. 6(b)) and at the fold was about $5.18 \mathrm{~nm}$ (Fig. 6(c)). 

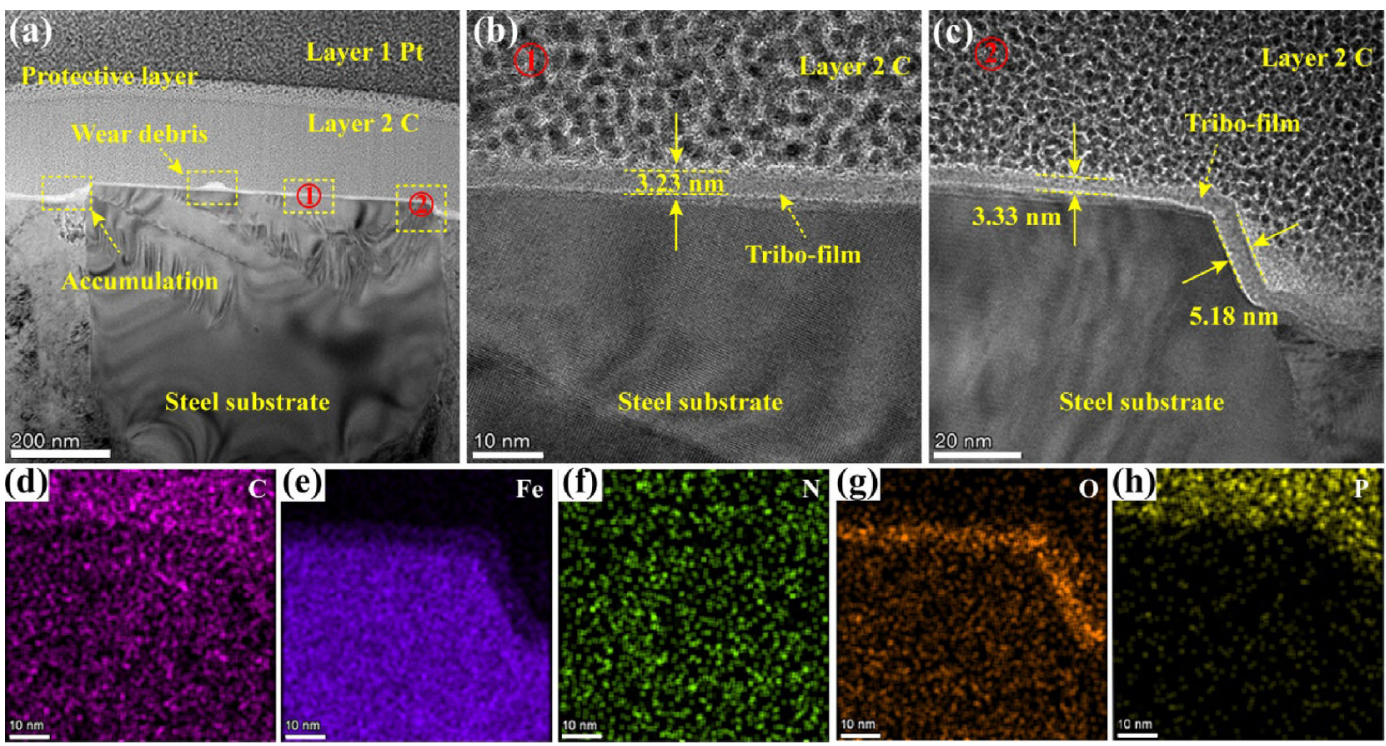

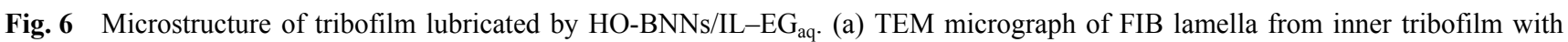
close-up view, (b, c) HRTEM images of yellow rectangular area at 1 and 2, respectively, and (d-h) EDS element mapping images of cross section of region 2 of $\mathrm{C}, \mathrm{Fe}, \mathrm{N}, \mathrm{O}$, and $\mathrm{P}$.

EDS element mapping (Figs. 6(d)-6(h)) suggested that the tribofilm was mainly composed of $\mathrm{C}, \mathrm{Fe}, \mathrm{N}$, $\mathrm{O}$, and $\mathrm{P}$. The $\mathrm{C}$ element originated from tribochemical reaction products between IL and EG, whereas the $\mathrm{N}$ element was from HO-BNNs and the decomposition products of the amino group of IL. The rich Fe and O elements corresponding to iron oxides were formed by the tribochemical reactions between the free oxygen or decomposition oxygen and the steel surface in the process of friction. This result is consistent with the XPS result.

Changes in the chemical composition and morphology of lubricants after friction can also help reveal the lubrication mechanism [46]. Figure 7 shows the microstructure of the wear debris collected from the residual liquid after friction. Zooming in on some of the typical regions, the structures with lattice spacings of $0.25 \mathrm{~nm}\left[\mathrm{Fe}_{3} \mathrm{O}_{4}(311)\right]$ and $0.33 \mathrm{~nm}$ [BNNs (002)] can be determined (Fig. 7(b)). The structures with lattice spacings of $0.21 \mathrm{~nm}\left[\mathrm{Fe}_{3} \mathrm{O}_{4}(110)\right]$ and $0.33 \mathrm{~nm}$ [BNNs (002)] can be detected in other regions (Fig. 7(c)) [39, $47,48]$. This was further confirmed by EDS mapping images (Fig. 7(d)). Compared with the initial high crystallization of HO-BNNs (Fig. 1(b)), the degree of crystallization of HO-BNNs was significantly reduced after friction. In addition, Fig. 7(f) shows very small quantum dot particles composed of $\mathrm{BN}$ that appear in the residual liquid, which may be generated from the continuous consumption of HO-BNNs. The corresponding height profiles (Fig. 7(g)) intuitively revealed that the average height of the quantum dots was $5.12 \pm 0.69 \mathrm{~nm}$. Moreover, Fig. 7(g) also reveals some large unconsumed HO-BNNs with a width of $1.7 \mu \mathrm{m}$ and height of $4.95 \mathrm{~nm}$. These results indicate that changes in HO-BNNS during the friction process included a reduction in size and a decrease in the degree of crystallization. These small BN particles can fill the furrow of the worn surface to repair the worn surface, further assisting in achieving superlubricity.

A segmented experiment was conducted at different time to obtain further insights into the evolution of macroscale superlubricity achieved by $\mathrm{HO}-\mathrm{BNNs} / \mathrm{IL}-$ $E_{\text {aq. }}$. Figure 8(a) shows the evolution of the COF and contact pressure at five status points $(115,375,1,200$, $1,800$, and $3,600 \mathrm{~s})$. The COF increased sharply to 0.21 within $50 \mathrm{~s}$. Then, the COF gradually decreased and entered the superlubricity area $(\mathrm{COF}<0.01)$ at approximately $600 \mathrm{~s}$. By contrast, the contact pressure decreased sharply from the initial value of $1.814 \mathrm{GPa}$ to $786 \mathrm{MPa}$ at state 1 within $115 \mathrm{~s}$. Subsequently, the contact pressure continually decreased to $499 \mathrm{MPa}$ in state 2 within $260 \mathrm{~s}$. After entering the superlubricity region, the contact pressure decreased slowly to $163 \mathrm{MPa}$ within 3,000 s. Based on the Stribeck theory 

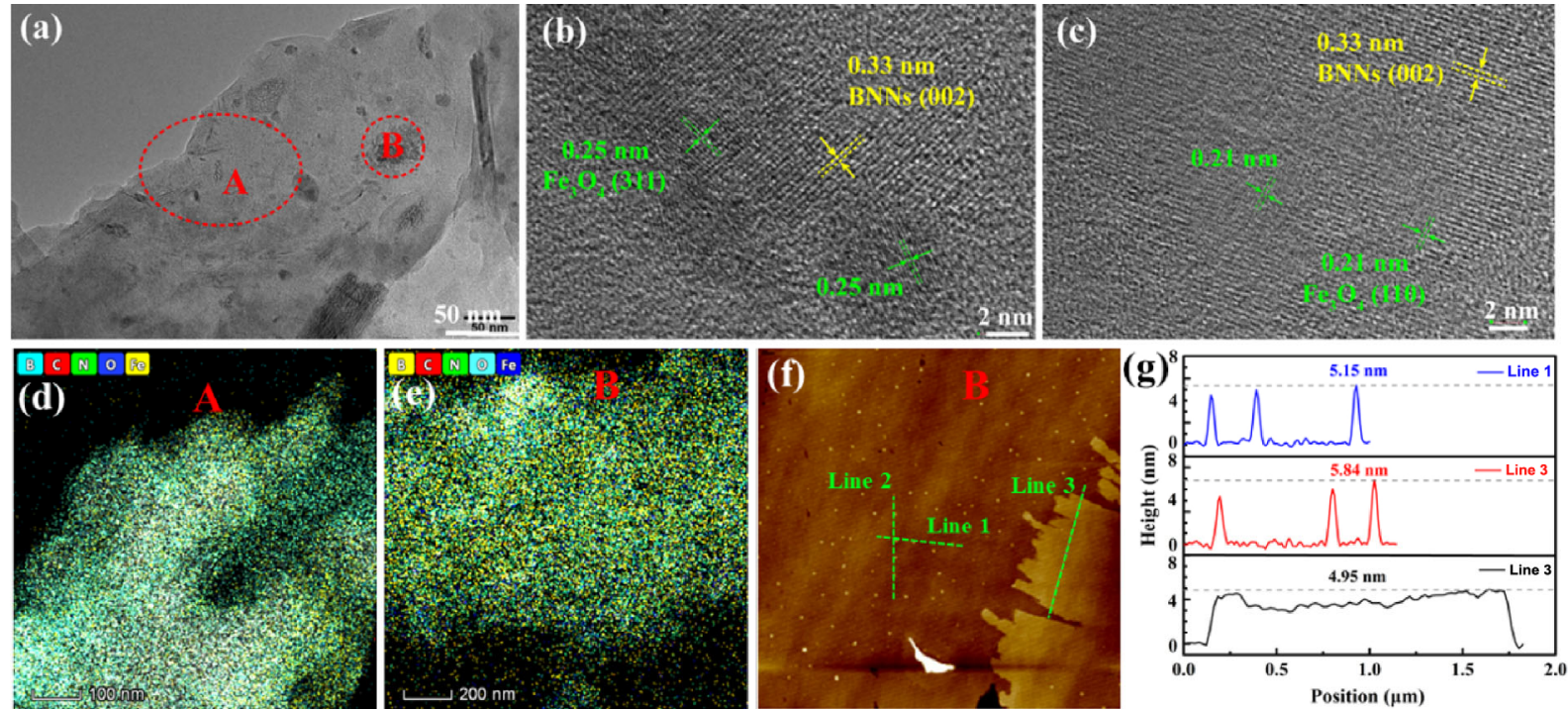

Fig. 7 Characteristics of wear debris collected from residual liquid of HO-BNNs/IL-EG aq after friction. (a) TEM images; (b, c) HRTEM images in two different regions, demonstrating typical $\mathrm{BN}$ and $\mathrm{Fe}_{3} \mathrm{O}_{4}$ structure; (d, e) EDS mapping images for different sites A and $\mathrm{B}$ corresponding to (a); (f) AFM height image of wear debris; (g) corresponding height profiles along green lines in (f); revealing decrease in HO-BNNs size after friction.
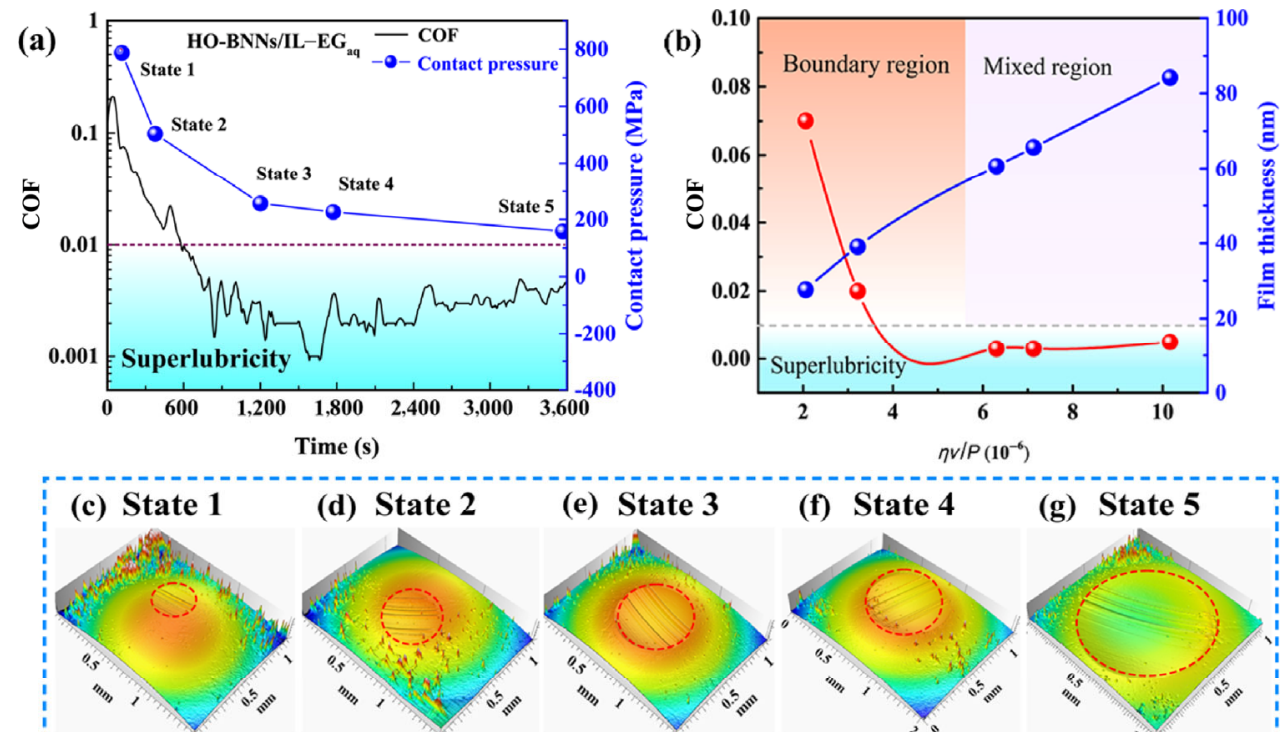

(d) State 2

(e) State 3

(f) State 4

(g) State 5
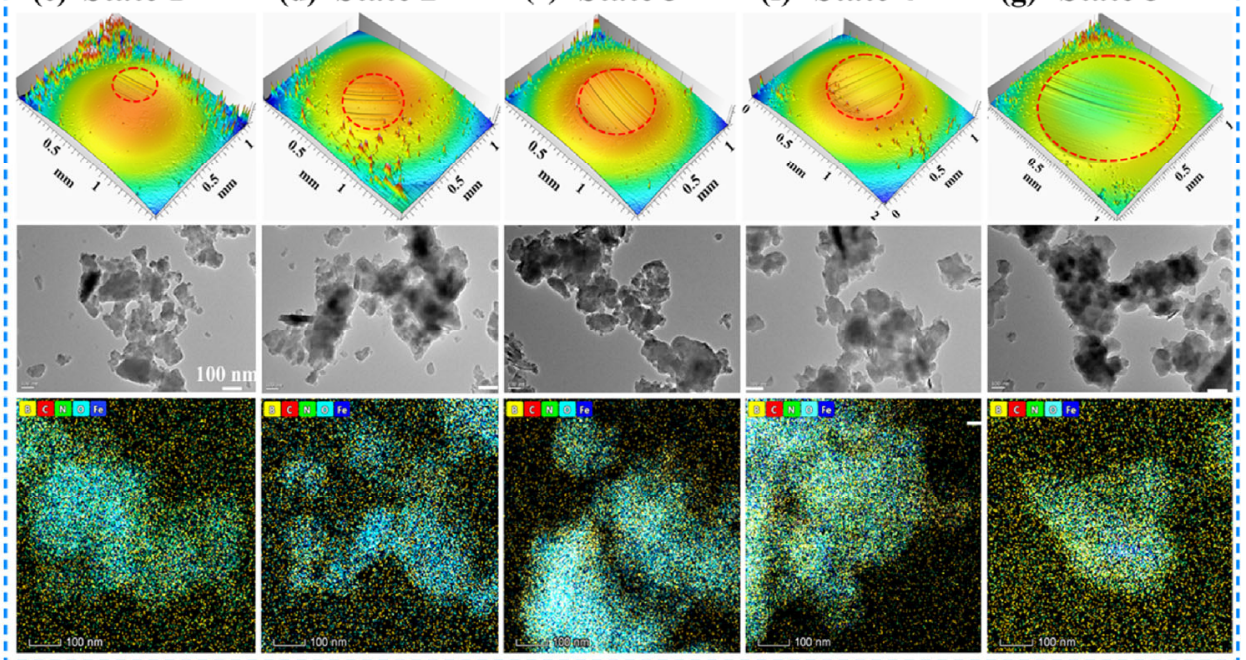

Fig. 8 (a) Evolution of COFs and contact pressures with time, (b) Stribeck curve and film thickness as function of Sommerfeld number $(\eta v / p)$ at different time, $(\mathrm{c}-\mathrm{g}) 3 \mathrm{D}$ micrographs of worn tracks, TEM images, and EDS mapping of wear debris collected from wear surfaces in different states (at 115, 375, 1,200, 1,800, and 3,600 s, respectively) lubricated by HO-BNNs/IL-EG aq at $196 \mathrm{~N}$ and 1,200 r/min. 
(Section 4 in the ESM) and surface roughness, the contact pressure $(\tau)$, theoretical film thickness $\left(h_{\mathrm{c}}\right)$, and film thickness ratio $(\lambda)$ at different stages were calculated, as shown in Table 1. During the running-in period (state 1 and state 2 as representative), $\lambda$ was approximately 0.30 and 0.85 , respectively, indicating that the lubrication was still in the boundary lubrication regime (Fig. 8(b)) $[49,50]$. In state 3 (i.e., at 1,200 s), $\lambda$ was approximately 1.27 , and the friction entered into the mixed lubrication regime. Subsequently, even though the superlubricity state could still be maintained (state 4 and state 5 as representative), $\lambda$ increased to 1.34 and 2.51, respectively, and the lubrication was still in the mixed lubrication regime. Therefore, the friction process lubricated by HO-BNNs/IL-EG $\mathrm{aq}_{\text {aq }}$ experienced boundary lubrication during the running-in period (nonsuperlubricity) and boundary lubrication and mixed lubrication (superlubricity).

To further study the evolution of the contact surface, the microstructure characterization of wear scars and wear debris at different states was performed by the SEM (Fig. S8 in the ESM), 3D micrographs, and TEM, as depicted in Figs. 8(c)-8(g). In state 1, the SEM images and $3 \mathrm{D}$ micrographs illustrated that there were many large exfoliations and severe pits on the contact surface owing to initial severe wear. Moreover, the TEM images of wear debris collected from the worn surfaces indicated that HO-BNNs almost maintained their original size, accompanied by some large iron filings. Then, in state 2 , the contact surface was very rough with many deep and wide grooves, which was typical abrasive wear. In addition, some small HO-BNNs with more iron filings were captured, and some HO-BNNs were ground to be amorphous during friction. Subsequently, as compared with superlubricity states 3, 4, and 5, some finer furrows were found and the worn surfaces became smoother over time. TEM

Table 1 Contact pressure $(\tau)$ and theoretical film thickness $\left(h_{\mathrm{c}}\right)$ of liquid lubricating film, roughness of contacting surface $(\sigma)$, and film thickness ratio $(\lambda)$.

\begin{tabular}{cccccc}
\hline Item & State 1 & State 2 & State 3 & State 4 & State 5 \\
\hline$\tau(\mathrm{MPa})$ & 786.5 & 503.3 & 256.9 & 227.1 & 159.2 \\
$h_{\mathrm{c}}(\mathrm{nm})$ & 48.2 & 62.2 & 86.6 & 91.5 & 110.4 \\
$\sigma(\mathrm{nm})$ & 160.5 & 73.2 & 68.4 & 68.1 & 44.1 \\
$\lambda$ & 0.30 & 0.85 & 1.27 & 1.34 & 2.51 \\
\hline
\end{tabular}

images of the wear debris indicated that the structure of HO-BNNs was destroyed to form a large amount of debris mixed with fewer iron filings. The decrease in the roughness of the contact surface changed the lubrication state. Therefore, the wear of friction pairs mainly occurred during the early violent running-in period. Furthermore, the contact surface became smoother over time owing to the polishing and selfrepairing effects of the HO-BNNs.

\subsection{Simulation of superlubricity system}

To further analyze the macroscale superlubricity mechanism of HO-BNNs/IL-EG ${ }_{\mathrm{aq}}$ dispersion, molecular dynamics (MD) simulations were performed. The electrostatic interaction (Coul) and van der Waals interaction (VDW) between the components of the dispersion and the steel substrate were calculated. Figure 9(a) shows a structure optimization diagram of the lubrication system built by VESTA and VMD (Section 5 in the ESM) according to the mass ratio of the components. Figure $9(\mathrm{~b})$ displays the interaction energies between the EG and other components. It is clear that the VDW interaction between EG and HOBNNs was approximately $-2,795 \mathrm{~kJ} / \mathrm{mol}$, which was the strongest. The interaction between EG and cations (CAI) is lighter, and the VDW interaction plays a more important role. Coul contributed more to the interaction between EG and anions (ANI). Furthermore, the interactions between water $(\mathrm{W})$ and other components were analyzed (Fig. 9(c)). The results showed that the Coul interaction between $\mathrm{W}$ and HO-BNNs was the strongest (approximately $-1,351 \mathrm{~kJ} / \mathrm{mol}$ ). For the interactions between $\mathrm{W}$ and CAI, both Coul and VDW were the lowest.

To determine the adsorption procedure on the metal surface, the interaction between Fe and the other components is shown in Fig. S9 in the ESM. The results show that the VDW interaction between Fe and EG was the strongest (approximately $-4,686 \mathrm{~kJ} / \mathrm{mol}$ ). For the interaction between Fe and ANI, CAI, and HO$\mathrm{BNNs}$, the VDW was relatively weak. However, the electrostatic interactions between Fe and EG, CAI, ANI, and HO-BNNs make almost no contribution.

The aforedescribed calculation results reveal the interaction among the components of the lubricant. First, EG molecules were adsorbed on the steel surface 

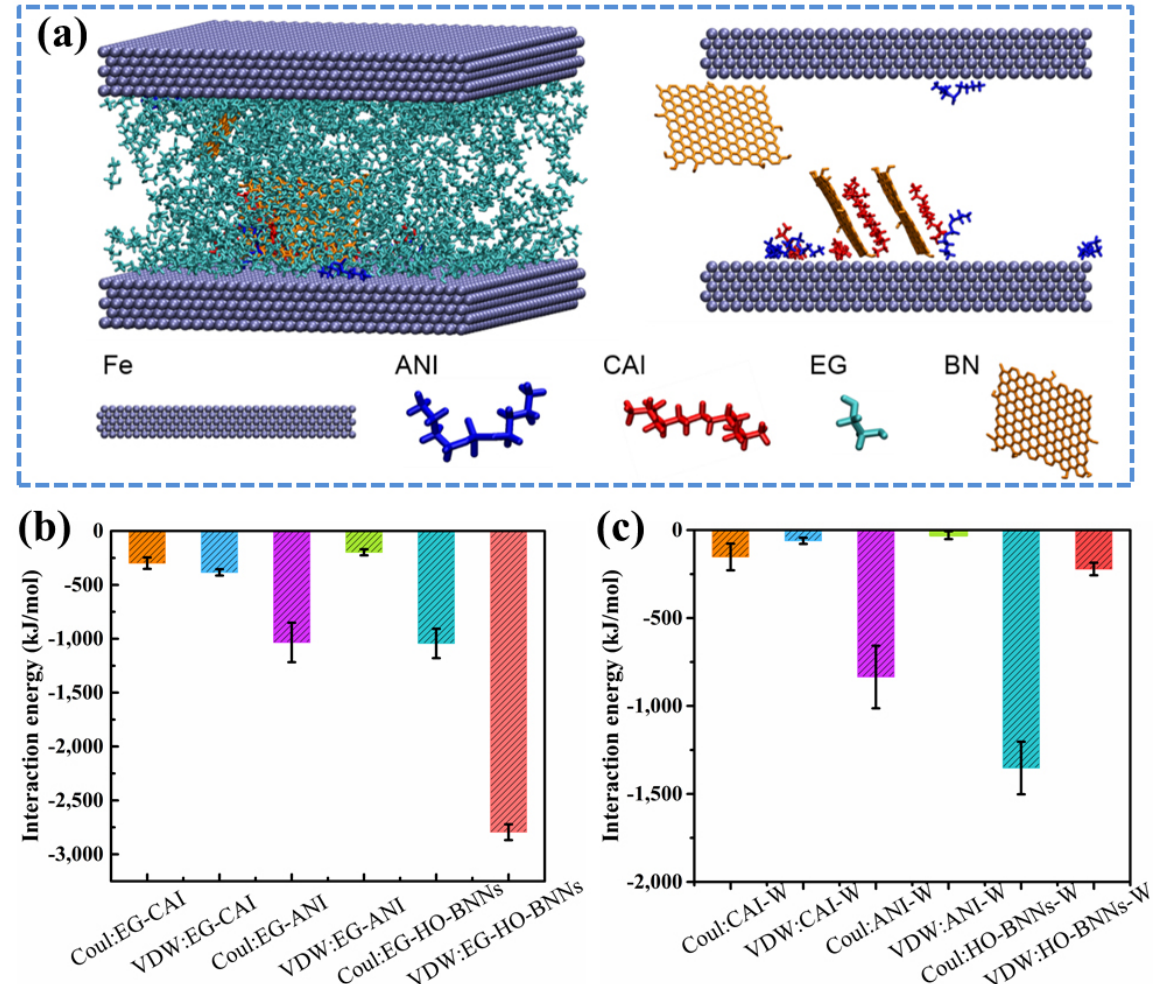

Fig. 9 (a) Snapshot of simulated system including Fe, anion, cation, EG, and HO-BNNs. Electrostatic interaction energy (Coul) and van der Waals interaction energy (VDW) among (b) EG and CAI (cation), ANI (anion), and HO-BNNs, and (c) water and CAI, ANI, and HO-BNNs.

owing to the strong VDW effect, which ensured that severe corrosion did not occur in the contact area. Second, a stronger VDW interaction between EG and HO-BNNs may prompt the HO-BNNs to adsorb to the second layer, which enables HO-BNNs to be ground during the rubbing process to polish and repair the worn surface. In addition, anions and EG have an inferior interaction, which indicates that the IL may be adsorbed to the third layer. ILs are also involved in tribochemical reactions to form a tribofilm. These conclusions help reveal the macroscale superlubricity mechanism of the system.

\subsection{Superlubricity mechanism}

The aforementioned results and analyses confirm that $\mathrm{HO}-\mathrm{BNNs} / \mathrm{IL}-\mathrm{EG}_{\mathrm{aq}}$ can achieve long-lasting and robust macroscale superlubricity between steel and steel during point-to-point contact. This can be explained mainly by the synergetic effects between IL and $\mathrm{HO}-\mathrm{BNN}$ in $\mathrm{EG}_{\mathrm{aq}}$. A feasible lubrication mechanism is shown in Fig. 10. In the initial state, a small amount of lubricant entered the gaps between the steel ball contacts. Then, EG molecules were first adsorbed onto the steel surface by VDW interaction to form a protective layer, as shown in Fig. 10(b), which ensured minimal corrosion. Moreover, IL adsorbed on the surface of EG molecules to form a regular distribution with a certain concentration gradient. This not only changes the shear stress of the lubricant but also participates in the tribochemical reaction to generate a tribofilm.

In addition, HO-BNNs were evenly dispersed in the gaps of the contact surface owing to hydrogen bonding. At the beginning of the friction test, boundary lubrication was dominant because of the extremely high initial contact pressure (approximately 1.8 GPa). During the running-in period, asperity wear occurred between the friction pairs (Fig. 10(d)), which led to a gradual reduction in the contact pressure. Meanwhile, EG molecules and the IL were further adsorbed on the fresh steel surface to form a tribofilm through a tribochemical reaction. The entrained HO-BNNs were crushed under extrusion, and then the fresh $\mathrm{HO}-$ BNNs were continuously delivered into the contact 


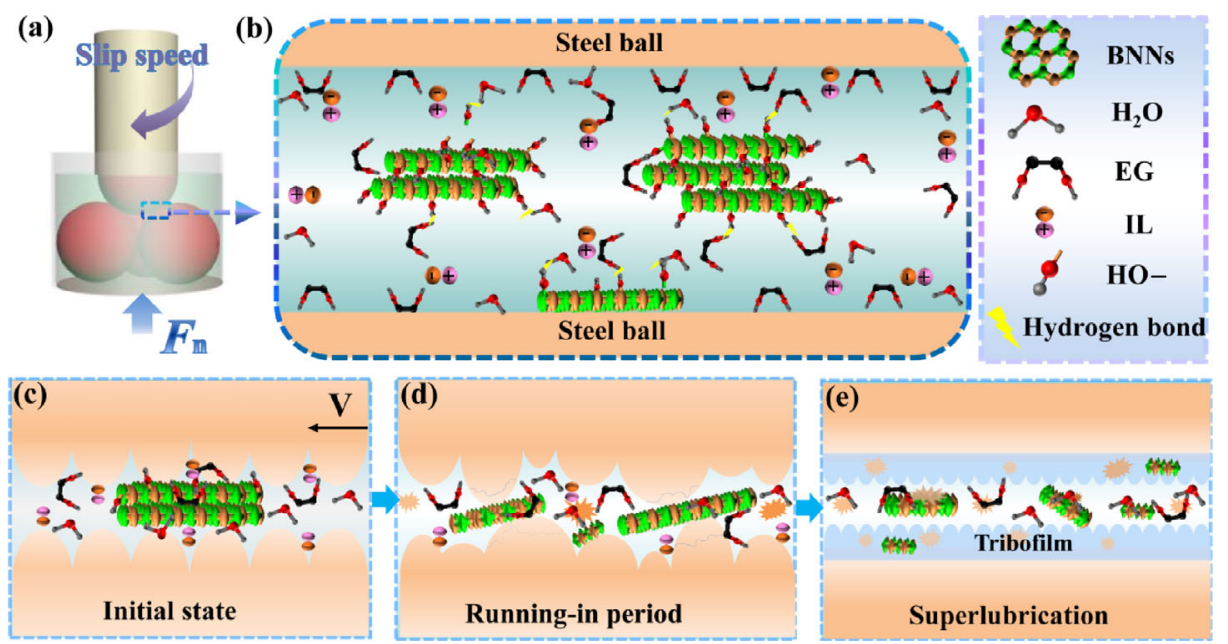

Fig. 10 (a) Schematic diagram of superlubricity system for steel-steel contacts, (b) schematic contact zone between steel-steel surfaces with lubrication of $\mathrm{HO}-\mathrm{BNNs} / \mathrm{IL}-\mathrm{EG}_{\mathrm{aq}}$ solution, containing adsorption of IL on steel ball surface and interaction between HO-BNNs and

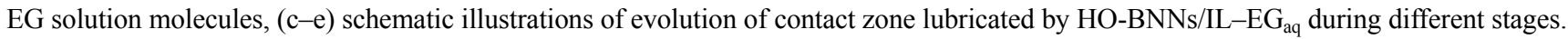

gaps. The high-affinity surface of these collapsed HO-BNNs adsorbed onto the steel surface assists in the mechanochemical reaction, surface polishing, and self-cleaning. Subsequently, as the tribofilm was continuously formed and polished by HO-BNNs, the friction entered into the mixed lubrication state, and finally a stable macroscale superlubricity state was achieved, as shown in Fig. 10(e). Furthermore, these flat tribofilms can avoid the metal asperity contact to reduce the surface pitting propagation and enhance the antiwear property.

\section{Conclusions}

A robust macroscale superlubricity for point-to-point contact on a steel interface was reported for the first time. Starting from two important factors of liquid superlubricity, namely, hydration and the running-in period, a proton-type IL was employed as a hydrated ion to adsorb onto the friction surfaces forming a hydration layer. HO-BNNs with good dispersion were prepared by a simple method and adopted as a polishing and self-repairing agent. The superlubricity state can be achieved in a suitable load range $(<250 \mathrm{~N})$ and sliding speed range $(>1,200 \mathrm{r} / \mathrm{min})$. In addition, EG can be substituted for other polyols to achieve superlubricity.

Through an analysis of the morphologies and elemental composition of worn surfaces, wear debris, and residual liquids, the following conclusions were obtained:

1) The hydrated ion generated from the proton-type IL played a vital role;

2) During the rubbing process, $\mathrm{HO}-\mathrm{BNNs}$ transformed from crystalline to amorphous form, and they were gradually consumed as the polishing and self-repairing agent to form quantum dots;

3) The worn surface can form a 3-5 nm thick tribofilm that is composed of abundant iron oxides, a small amount of HO-BNNs wear debris, and the reactants between IL and iron. State experiments revealed that the lubrication state changed from an initial boundary lubrication to mixed lubrication.

This superlubricity system can be directly applied to engineering applications. In addition, the design idea of this lubricant can be used as a reference for the development of other superlubricity systems. In addition, this has significant application prospects in other fields because it is green, cheap, and sustainable.

\section{Acknowledgements}

The authors are gratefully for the financial support provided by the Youth Innovation Promotion Association CAS (2021422).

Electronic Supplementary Material Supplementary material is available in the online version of this 
article at https://doi.org/10.1007/s40544-021-0545-x.

Open Access This article is licensed under a Creative Commons Attribution 4.0 International License, which permits use, sharing, adaptation, distribution and reproduction in any medium or format, as long as you give appropriate credit to the original author(s) and the source, provide a link to the Creative Commons licence, and indicate if changes were made.

The images or other third party material in this article are included in the article's Creative Commons licence, unless indicated otherwise in a credit line to the material. If material is not included in the article's Creative Commons licence and your intended use is not permitted by statutory regulation or exceeds the permitted use, you will need to obtain permission directly from the copyright holder.

To view a copy of this licence, visit http://creativecommons.org/licenses/by/4.0/.

\section{References}

[1] Holmberg K, Erdemir A. Influence of tribology on global energy consumption, costs and emissions. Friction 5(3): 263-284 (2017)

[2] Cai M, Yu Q, Liu W, Zhou F. Ionic liquid lubricants: When chemistry meets tribology. Chem Soc Rev 49(21): 7753-7818 (2020)

[3] Meng Y G, Xu J, Jin Z M, Prakash B, Hu Y Z. A review of recent advances in tribology. Friction 8(2): 221-300 (2020)

[4] Luo J B, Zhou X. Superlubricitive engineering-Future industry nearly getting rid of wear and frictional energy consumption. Friction 8(4): 643-665 (2020)

[5] Kawai S, Benassi A, Gnecco E, Söde H, Pawlak R, Feng X, Müllen K, Passerone D, Pignedoli CA, Ruffieux P, et al. Superlubricity of graphene nanoribbons on gold surfaces. Science 351(6276): 957-961 (2016)

[6] Wang H D, Liu Y H. Superlubricity achieved with twodimensional nano-additives to liquid lubricants. Friction 8(6): 1007-1024 (2020)

[7] Goldberg R, Schroeder A, Silbert G, Turjeman K, Barenholz $\mathrm{Y}$, Klein J. Boundary lubricants with exceptionally low friction coefficients based on 2D close-packed phosphatidylcholine liposomes. Adv Mater 23(31): 3517-3521 (2011)

[8] Zhai W Z, Zhou K. Nanomaterials in superlubricity. Adv Funct Mater 29(28): 1806395 (2019)

[9] Seror J, Zhu L, Goldberg R, Day AJ, Klein J. Supramolecular synergy in the boundary lubrication of synovial joints. Nat Commun 6: 6497 (2015)
[10] Chen M, Briscoe WH, Armes SP, Klein J. Lubrication at physiological pressures by polyzwitterionic brushes. Science 323(5922): 1698-1701 (2009)

[11] Li P P, Ju P F, Ji L, Li H X, Liu X H, Chen L, Zhou H D, Chen J M. Toward robust macroscale superlubricity on engineering steel substrate. Adv Mater 32(36): 2002039 (2020)

[12] Chen X C, Zhang C H, Kato T, Yang X N, Wu S D, Wang R, Nosaka M, Luo J B. Evolution of tribo-induced interfacial nanostructures governing superlubricity in a-C:H and a-C:H:Si films. Nat Commun 8: 1675 (2017)

[13] Liu S W, Wang H P, Xu Q, Ma T B, Yu G, Zhang C H, Geng D C, Yu Z W, Zhang S G, Wang W Z, et al. Robust microscale superlubricity under high contact pressure enabled by graphene-coated microsphere. Nat Commun 8: 14029 (2017)

[14] Li J J, Gao T Y, Luo J B. Superlubricity of graphite induced by multiple transferred graphene nanoflakes. Adv Sci 5(3): 1700616 (2018)

[15] Chen X C, Li J J. Superlubricity of carbon nanostructures. Carbon 158: 1-23 (2020)

[16] Wu S C, Meng Z S, Tao X M, Wang Z. Superlubricity of molybdenum disulfide subjected to large compressive strains. Friction, 10(2): 209-216 (2022)

[17] Liu Y H, Chen L, Zhang B, Cao Z Y, Shi P F, Peng Y, Zhou N N, Zhang J Y, Qian L M. Key role of transfer layer in load dependence of friction on hydrogenated diamond-like carbon films in humid air and vacuum. Materials 12(9): 1550 (2019)

[18] Saravanan P, Selyanchyn R, Tanaka H, Darekar D, Staykov A, Fujikawa S, Lyth SM, Sugimura J. Macroscale superlubricity of multilayer polyethylenimine/graphene oxide coatings in different gas environments. ACS Appl Mater Interfaces 8(40): 27179-27187 (2016)

[19] Li K, Zhang S M, Liu D S, Amann T, Zhang C H, Yuan C Q, Luo J B. Superlubricity of 1,3-diketone based on autonomous viscosity control at various velocities. Tribol Int 126: 127-132 (2018)

[20] Qi S S, Geng Z R, Lu Z B, Zhang G G, Wu Z G. Synergistic lubricating behaviors of $3 \mathrm{D}$ graphene and $2 \mathrm{D}$ hexagonal boron nitride dispersed in $\mathrm{PAO}_{4}$ for steel/steel contact. $A d v$ Mater Interfaces 7(8): 1901893 (2020)

[21] Han T Y, Zhang C H, Luo J B. Macroscale superlubricity enabled by hydrated alkali metal ions. Langmuir 34(38): 11281-11291 (2018)

[22] Xu J, Li J J. New achievements in superlubricity from international workshop on superlubricity: Fundamental and applications. Friction 3(4): 344-351 (2015) 
[23] Liu L C, Zhou M, Jin L, Li L C, Mo Y T, Su G S, Li X, Zhu $\mathrm{H}$ W, Tian Y. Recent advances in friction and lubrication of graphene and other 2D materials: Mechanisms and applications. Friction 7(3): 199-216 (2019)

[24] $\mathrm{Xu} \mathrm{J} \mathrm{G,} \mathrm{Kato} \mathrm{K.} \mathrm{Formation} \mathrm{of} \mathrm{tribochemical} \mathrm{layer} \mathrm{of}$ ceramics sliding in water and its role for low friction. Wear 245(1-2): 61-75 (2000)

[25] Chen M, Kato K, Adachi K. The comparisons of sliding speed and normal load effect on friction coefficients of self-mated $\mathrm{Si}_{3} \mathrm{~N}_{4}$ and $\mathrm{SiC}$ under water lubrication. Tribol Int 35(3): 129-135 (2002)

[26] Raviv U, Klein J. Fluidity of bound hydration layers. Science 297(5586): 1540-1543 (2002)

[27] Briscoe W H, Titmuss S, Tiberg F, Thomas R K, McGillivray D J, Klein J. Boundary lubrication under water. Nature 444(7116): 191-194 (2006)

[28] Li J J, Zhang C H, Deng M M, Luo J B. Reduction of friction stress of ethylene glycol by attached hydrogen ions. Sci Rep 4: 7226 (2014)

[29] Ge X, Li J, Luo R, Zhang C, Luo J. Macroscale superlubricity enabled by the synergy effect of graphene-oxide nanoflakes and ethanediol. ACS Appl Mater Interfaces 10(47): 4086340870 (2018)

[30] Ge X, Li J, Zhang C, Wang Z, Luo J. Superlubricity of 1-ethyl-3-methylimidazolium trifluoromethanesulfonate ionic liquid induced by tribochemical reactions. Langmuir 34(18): 5245-5252 (2018)

[31] Jia W P, Tian J M, Bai P P, Li S W, Zeng H B, Zhang W L, Tian Y. A novel comb-typed poly(oligo(ethylene glycol) methylether acrylate) as an excellent aqueous lubricant. J Colloid Interface Sci 539: 342-350 (2019)

[32] Yi S, Li J J, Liu Y F, Ge X Y, Zhang J, Luo J B. In-situ formation of tribofilm with $\mathrm{Ti}_{3} \mathrm{C}_{2} \mathrm{~T}_{x}$ MXene nanoflakes triggers macroscale superlubricity. Tribol Int 154: 106695 (2021)

[33] Ma W, Gong Z B, Gao K X, Qiang L, Zhang J Y, Yu S R. Superlubricity achieved by carbon quantum dots in ionic liquid. Mater Lett 195: 220-223 (2017)

[34] Jahn S, Klein J. Hydration lubrication: The macromolecular domain. Macromolecules 48(15): 5059-5075 (2015)

[35] Amann T, Kailer A. Relationship between ultralow friction of mesogenic-like fluids and their lateral chain length. Tribol Lett 41(1): 121-129 (2011)

[36] Ren X Y, Yang X, Xie G X, He F, Wang R, Zhang C H, Guo D, Luo J B. Superlubricity under ultrahigh contact pressure enabled by partially oxidized black phosphorus nanosheets. npj 2D Mater Appl 5: 44 (2021)

[37] Ma L R, Gaisinskaya-Kipnis A, Kampf N, Klein J. Origins of hydration lubrication. Nat Commun 6(1): 6060 (2015)
[38] Zhang Y Z, Kovalev A, Meng Y G. Combined effect of boundary layer formation and surface smoothing on friction and wear rate of lubricated point contacts during normal running-in processes. Friction 6(3): 274-288 (2018)

[39] Pakdel A, Bando Y, Golberg D. Nano boron nitride flatland. Chem Soc Rev 43(3): 934-959 (2014)

[40] Bai Y F, Wang L X, Ge C H, Liu R, Guan H Y, Zhang X D. Atomically thin hydroxylation boron nitride nanosheets for excellent water-based lubricant additives. J Am Ceram Soc 103(12): 6951-6960 (2020)

[41] Wang L X, Han W F, Ge C H, Zhang R, Bai Y F, Zhang $\mathrm{X}$ D. Covalent functionalized boron nitride nanosheets as efficient lubricant oil additives. Adv Mater Interfaces 6(21): 1901172 (2019)

[42] Zhang R, Ding Q, Zhang S, Niu Q, Ye J, Hu L. Construction of a continuously layered structure of h-BN nanosheets in the liquid phase via sonication-induced gelation to achieve low friction and wear. Nanoscale 11(26): 12553-12562 (2019)

[43] Bai C N, An L L, Zhang J, Zhang X K, Zhang B, Qiang L, Yu Y L, Zhang J Y. Superlow friction of amorphous diamond-like carbon films in humid ambient enabled by hexagonal boron nitride nanosheet wrapped carbon nanoparticles. Chem Eng J 402: 126206 (2020)

[44] Chen Y, Zhao D, Qi F, Liu W W. Scratch behavior of boron nitride nanotube/boron nitride nanoplatelet hybrid reinforced $\mathrm{ZrB}_{2}-\mathrm{SiC}$ composites. Ceram Int 44(3): 3277-3281 (2018)

[45] Li K, Amann T, Walter M, Moseler M, Kailer A, Rühe J. Ultralow friction induced by tribochemical reactions: A novel mechanism of lubrication on steel surfaces. Langmuir 29(17): 5207-5213 (2013)

[46] Ge X Y, Li J J, Wang H D, Zhang C H, Liu Y H, Luo J B. Macroscale superlubricity under extreme pressure enabled by the combination of graphene-oxide nanosheets with ionic liquid. Carbon 151: 76-83 (2019)

[47] Liang B Y, Zhang L J, Zhang W X, Zhang T C, Zhang J Z, Jiao M L, Yang L. BN/Fe hybrid composites as highly efficient visible-light-driven photocatalysts. J Inorg Organomet Polym Mater 30(5): 1634-1642 (2020)

[48] Pang H, Pang W H, Zhang B, Ren N. Excellent microwave absorption properties of the h-BN-GO- $\mathrm{Fe}_{3} \mathrm{O}_{4}$ ternary composite. J Mater Chem C 6(43): 11722-11730 (2018)

[49] Jia W, Bai P, Zhang W, Ma L, Meng Y, Tian Y. On lubrication states after a running-in process in aqueous lubrication. Langmuir 35(48): 15435-15443 (2019)

[50] Ge X Y, Halmans T, Li J J, Luo J B. Molecular behaviors in thin film lubrication-Part three: Superlubricity attained by polar and nonpolar molecules. Friction 7(6): 625-636 (2019) 


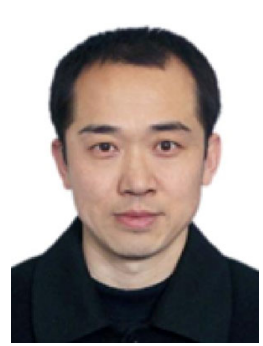

Dapeng FENG. He received his Ph.D. degree in physical chemistry in 2001 from Lanzhou Institute of Chemical Physics, Chinese Academy of Sciences (CAS). He is a professor at the State Key Lab of Solid Lubrication in Lanzhou Institute

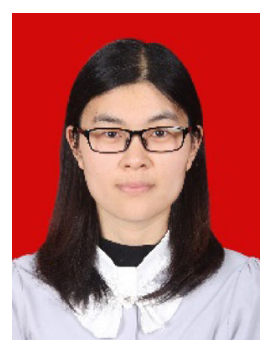

Dan QIAO. She received her Ph.D. degree in material science in 2014 from the Lanzhou Institute of Chemical Physics, CAS. She is an associate professor at the State

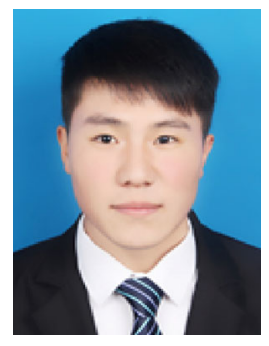

Zhiwen ZHENG. He received his B.S. degree from the China University of Mining and Technology, 2016. $\mathrm{He}$ got his M.S. degree at the Lanzhou Institute of Chemical Physics, CAS, China, 2019. Now, he

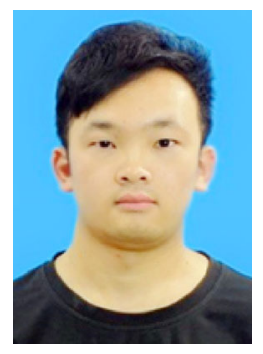

Xiaolong LIU. He received his M.S. degree in 2019 from the Shandong Normal University. After graduation, he was employed by Lanzhou

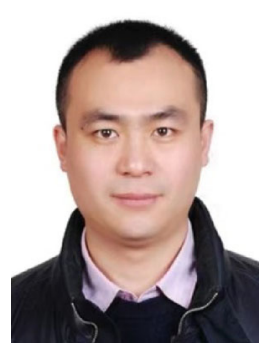

Guowei HUANG. He received his Ph.D. degree in 2017 from the State Key Laboratory of solid lubrication, Lanzhou Institute of Chemical Physics, CAS. Now, he is a research of Chemical Physics, CAS. He has authored or coauthored more than 80 journal papers. His research interests are high performance lubricating oil and grease and additives. He has gained a number of awards including "Second Prize of National Technical Invention" and "Technology Invention Award of Gansu Province".

Key Laboratory of Solid Lubrication in the Lanzhou Institute of Chemical Physics, CAS. She has authored or co-authored more than 30 journal papers. Her research interests are ionic liquids lubricants and tribology chemistry.

continues to study for a Ph.D. degree in the Lanzhou Institute of Chemical Physics, CAS. His current scientific interests are devoted to macroscale liquid superlubricity and novel environmentally lubricating additives.

Institute of Chemical Physics, CAS. His scientific interests are mainly focused on the design, preparation, and tribo-chemistry of high-performance lubricant.

assistant at Lanzhou University of Technology. His research interests include the design and preparation of novel ionic liquids as lubricant and lubricating additives. 


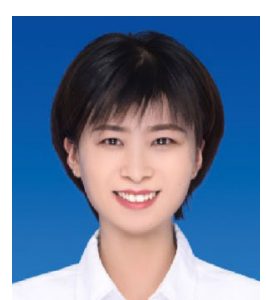

Haijie CHEN. She is a Ph.D. student at the Lanzhou Institute of
Chemical Physics, CAS. Her ongoing research focuses on assembly lubrication film of 2D materials.

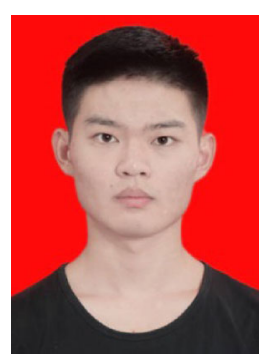

Hongxiang YU. He received his B.S. degree in 2018 from the Shandong Normal University. Now, he is studying for a Ph.D. degree in
Lanzhou Institute of Chemical Physics, CAS. His current scientific interests are devoted to mechanism and kinetic analysis of tribochemical reaction of lubricants, as well as novel lubricant additives. 